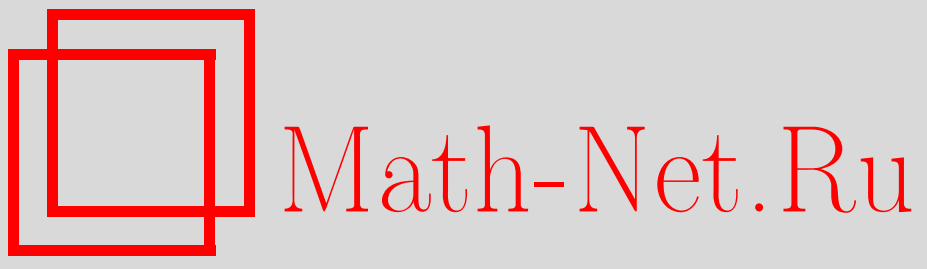

Д. А. Попов, Обобщенное преобразование Радона на плоскости, его обращение и условия Кавальери, Функи. анализ и его прил., 2001, том 35, выпуск 4, 38-53

DOI: https://doi.org/10.4213/faa272

Использование Общероссийского математического портала MathNet.Ru подразумевает, что вы прочитали и согласны с пользовательским соглашением

http://www . mathnet.ru/rus/agreement

Параметры загрузки:

IP : 54.198 .187 .58

26 апреля 2023 г., 15:10:28

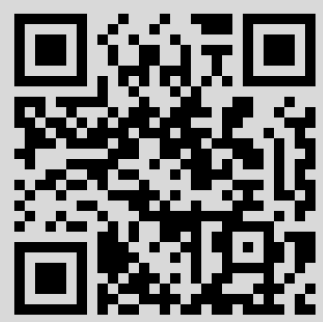




\title{
Обобщенное преобразование Радона на плоскости, его обращение и условия Кавальери*
}

\author{
(c) 2001. Д. А. Попов
}

\section{§1. Введение}

Пусть $\mathbb{D}-$ открытый единичный круг в $\mathbb{R}^{2}$ с координатами $x=\left(x_{1}, x_{2}\right), \overline{\mathbb{D}}-$ его замыкание и $\partial \mathbb{D}-$ граница. Преобразование Радона $\mathscr{R} u$ функции $u: \mathbb{R}^{2} \rightarrow \mathbb{R}$ с носителем в $\mathbb{D}$ задается равенством

$$
\mathscr{R} u(\varphi, q) \equiv J(\varphi, q)=\int_{\gamma_{0}(\varphi, q)} u d s=\int_{-\sqrt{1-q^{2}}}^{\sqrt{1-q^{2}}} u\left(x^{0}(t)\right) d t,
$$

где $\gamma_{0}(\varphi, q)$ - лежащий внутри $\overline{\mathbb{D}}$ отрезок прямой $(\varphi, q)$, задаваемой уравнением $x=x^{0}(t)=q \eta-t \eta^{*}$. Здесь и ниже $\eta=(\cos \varphi, \sin \varphi), \eta^{*}=(-\sin \varphi, \cos \varphi)$. Преобразование Радона обратимо $[1-4]$, и при $u \in L^{2}\left(\mathbb{R}^{2}\right)$

$$
u(x)=\left(\mathscr{R}^{-1} J\right)(x)=\frac{1}{4 \pi^{2}} \int_{0}^{\infty} \lambda d \lambda \int_{0}^{2 \pi} e^{-i \lambda\langle x, \eta\rangle} \widehat{J}(\varphi, \lambda) d \varphi .
$$

В этой формуле и далее в тексте $\langle\cdot, \cdot\rangle$ - скалярное произведение в $\mathbb{R}^{2},|x|-$ норма в $\mathbb{R}^{2}$ и $\widehat{J}, \hat{u}-$ преобразования Фурье,

$$
\widehat{J}(\varphi, \lambda)=\int_{-\infty}^{+\infty} e^{i \lambda q} J(\varphi, q) d q, \quad \hat{u}(\xi)=\int e^{i\langle\xi, x\rangle} u(x) d x .
$$

Здесь и ниже $\int \cdots d x, \int \cdots d \xi$ означает интегрирование по $\mathbb{R}^{2}$. В настоящей работе рассматривается вопрос об обращении обобщенного преобразования $\mathrm{Pa}$ дона, при определении которого хорды $\gamma_{0}(\varphi, q)$ в (1.1) заменяются близкими к ним отрезками кривых $\gamma_{z}(\varphi, q)$. Кривая $\gamma_{z}(\varphi, q)$ будет задаваться уравнениями

$$
x=x(t)=x^{0}(t)+z(\varphi, q, t)=q \eta-t \eta^{*}+z(\varphi, q, t),
$$

и предполагается, что векторные поля $z(\varphi, q, t)$ удовлетворяют условиям

$$
\begin{gathered}
z(\varphi+\pi,-q,-t)=z(\varphi, q, t), \quad z\left(\varphi, q, \pm \sqrt{1-q^{2}}\right)=0, \\
x(t) \in \mathbb{D} \quad\left(|t|<\sqrt{1-q^{2}}\right) .
\end{gathered}
$$

Изменяя, если нужно, параметр $t$, можно считать, что $\left\langle z, \eta^{*}\right\rangle=0$, но мы не будем этим пользоваться. 
Таким образом, обобщенное преобразование Радона (ОПР) для функций с носителем в $\mathbb{D}$ определяется равенствами

$$
\begin{gathered}
\left(\mathscr{R}_{z} u\right)(\varphi, q) \equiv I(\varphi, q)=\int_{\gamma_{z}(\varphi, q)} u d s=\int_{-\sqrt{1-q^{2}}}^{\sqrt{1-q^{2}}} u(x(t))\left|\frac{d x}{d t}\right| d t \\
\left|\frac{d x}{d t}\right|=\left[\left(\frac{\partial x_{1}}{\partial t}\right)^{2}+\left(\frac{\partial x_{2}}{\partial t}\right)^{2}\right]^{1 / 2}=\left[1+\left|\frac{d z}{d t}\right|^{2}-2\left\langle\frac{d z}{d t}, \eta^{*}\right\rangle\right]^{1 / 2} .
\end{gathered}
$$

Задача состоит в восстановлении функции $u: \overline{\mathbb{D}} \rightarrow \mathbb{R}$, если известны величины $I(\varphi, q)$. Условия $(1.5)$ гарантируют, что $I(\varphi, q)$ - функция на пространстве $\mathbb{P}_{1}$ прямых, пересекающих $\overline{\mathbb{D}}$. При этом будет предполагаться, что векторные поля $z(\varphi, q, t)$ «достаточно малы» в подходящей метрике (см. ниже). Естественное требование на сеть кривых $\left\{\gamma_{z}(\varphi, q)\right\}$ - это условие ее регулярности, состоящее в том, что эта сеть топологически устроена как сеть прямых $\left\{\gamma_{0}(\varphi, q)\right\}$.

Рассматриваемая задача является двумерным вариантом общей задачи интегральной геометрии, в которой речь идет о восстановлении функции $f \in C_{0}^{\infty}\left(\mathbb{R}^{n}\right)$ по ее интегралам $\mathscr{R}_{M} f$ вдоль подмногообразий $M \subset \mathbb{R}^{n}$, зависящих от $n$ параметров. Геометрическая формулировка этой задачи была дана в работах [5-7]. После работы [6] стало ясно, что задачу обращения оператора $\mathscr{R}_{M}$ естественно рассматривать в рамках теории интегральных операторов Фурье (ИОФ). В частности, в этой работе показано, что при некоторых дополнительных условиях оператор $\mathscr{R}_{M}$ и его сопряженный $\mathscr{R}_{M}^{T}$ являются ИОФ и $\mathscr{R}_{M}^{T} \mathscr{R}_{M}-$ эллиптический псевдодифференциальный оператор (ПДО). Символ оператора $\mathscr{R}_{M}^{T} \mathscr{R}_{M}$ вычислен в работе [8], где решена задача обращения оператора $\mathscr{R}_{M}$ в предположении его трансляционной инвариантности. С различными аспектами теории ОПР в их связи с теорией ИОФ можно ознакомиться по работам [9-17]. Что касается задачи обращения, то, по-видимому, все известные результаты отвечают задачам, допускающим достаточно большую группу симметрий. Это относится и к классическим задачам, рассмотренным в $[1-4,18]$, задаче Куранта $[19,20]$, центральносимметричным задачам, рассмотренным в $[22,27]$, и к ряду других задач. Кроме задачи обращения в интегральной геометрии рассматривается задача описания образа оператора $\mathscr{R}_{M}$. Для оператора $\mathscr{R}_{z}$ в этом случае речь идет об условиях, при которых функция $I: \mathbb{P}_{1} \rightarrow \mathbb{R}$ представима в виде $I=\mathscr{R}_{z} u$. Следуя И. М. Гельфанду, будем называть эти условия условиями Кавальери. Для двумерного преобразования Радона они имеют вид

$$
\int_{-\infty}^{+\infty} q^{k} J(\varphi, q) d q=\sum_{l+m=k} q_{l m}(\cos \varphi)^{l}(\sin \varphi)^{m} .
$$

Ответ на вопрос об их достаточности для заданного класса функций $u: \mathbb{R}^{2} \rightarrow \mathbb{R}$ составляет содержание теорем типа Пэли-Винера $[1-4,23]$. В задачах без симметрий условия Кавальери, по-видимому, до сих пор получены не были.

Задаче обращения преобразования $\mathscr{R}_{z}$ был посвящен ряд работ математиков Новосибирской школы. В работе [24] в предположении регулярности сети $\left\{\gamma_{z}(\varphi, q)\right\}$ доказана теорема единственности $\left(\mathscr{R}_{z} u=0 \Longrightarrow u=0\right)$ и устойчивости задачи обращения. В работе [25] решена локальная задача восстановления функции по ее интегралам вдоль геодезических заданной конформно-плоской 
метрики. Локальность здесь означает, что задача решается в малой окрестности заданной точки $x_{0} \in \partial \mathbb{D}$. Условия Кавальери в этом случае не возникают. Среди задач интегральной геометрии в круге $\overline{\mathbb{D}}$ наибольший интерес представляет нелинейная задача восстановления конформно-плоской метрики по длинам геодезических с концами на $\partial \mathbb{D}$. В прикладной математике эта задача известна как основная кинематическая задача сейсмики, или задача ультразвуковой томографии. Пока в этой задаче получены только теоремы единственности и устойчивости [26] и локальное решение [27].

Автор благодарен Л. Р. Волевичу за полезные обсуждения.

\section{§2. Определения и предварительные сведения}

Везде ниже используется соглашение о константах: все константы обозначаются буквой $C$ и всегда указывается, от каких параметров они зависят, все константы эффективны в том смысле, что метод их вычисления содержится в доказательствах, и одной буквой могут обозначаться различные константы.

Будем предполагать, что векторные поля $z(\varphi, q, t)$ определены не только при $q^{2}+t^{2} \leqslant 1$, но и в более широкой области, например при $q^{2}+t^{2} \leqslant 4$. Умножая, если нужно, $z(\varphi, q, t)$ на гладкую срезающую функцию $\rho(q, t)(\rho(q, t)=1$ при $q^{2}+t^{2} \leqslant 1, \rho(q, t)=0$ при $\left.q^{2}+t^{2} \geqslant 4\right)$, будем всегда считать, что поля $z \equiv$ $z(\varphi, q, t)$ определены при всех $q, t$ и $z=0$ при $q^{2}+t^{2} \geqslant 4$. По определению $z=\left(z_{1}, z_{2}\right) \in\{z\}_{m}$, если конечна норма $\|z\|_{m}$,

$$
\|z\|_{m}=\sum_{0 \leqslant|\alpha| \leqslant m} \max _{(\varphi, q, t)}\left\{\left|\partial^{\alpha} z_{1}\right|+\left|\partial^{\alpha} z_{2}\right|\right\} \quad\left(\partial^{\alpha}=\partial_{\varphi}^{\alpha_{1}} \partial_{q}^{\alpha_{2}} \partial_{t}^{\alpha_{3}},|\alpha|=\alpha_{1}+\alpha_{2}+\alpha_{3}\right) .
$$

В данной работе рассматривается $L^{2}$-теория. Функции $u: \overline{\mathbb{D}} \rightarrow \mathbb{R}$ всегда считаются продолженными нулем на $\mathbb{R}^{2}$, и запись $u \in L^{2}\left(\mathbb{D}^{\prime}\right)$ означает, что $u \in L^{2}\left(\mathbb{R}^{2}\right)$ и $\operatorname{supp} u \subset \overline{\mathbb{D}}^{\prime} \subseteq \overline{\mathbb{D}}$. Нормы функций и операторов $A\left(A: L^{2}\left(\mathbb{R}^{2}\right) \rightarrow L^{2}\left(\mathbb{R}^{2}\right)\right)$ обозначаются через $\|u\|,\|A\|$, и $(u, v)$ - скалярное произведение в $L^{2}\left(\mathbb{R}^{2}\right)$. Через $\widetilde{\mathbb{P}}$ обозначается цилиндр $S^{1} \times \mathbb{R}$ с координатами $(\varphi, q)$ и $\mathbb{P}=\widetilde{\mathbb{P}} /[\sim]-$ пространство прямых в $\mathbb{R}^{2}$ (факторпространство цилиндра $\widetilde{\mathbb{P}}$ по отношению эквивалентности $(\varphi+\pi,-q) \sim(\varphi, q))$. Соответственно $\widetilde{\mathbb{P}}_{1}=S^{1} \times[-1,1]$ и $\mathbb{P}_{1}=\widetilde{\mathbb{P}}_{1} /[\sim]-$ пространство прямых, пересекающих $\overline{\mathbb{D}}$. В пространстве комплекснозначных функций на $\widetilde{\mathbb{P}}_{1}$ структура гильбертова пространства $L^{2}\left(\widetilde{\mathbb{P}}_{1}\right) \equiv L^{2}\left(\widetilde{\mathbb{P}}_{1}, \sqrt{1-q^{2}}\right)$ вводится с помощью скалярного произведения

$$
\left(F_{1}, F_{2}\right)_{L^{2}\left(\widetilde{\mathbb{P}}_{1}\right)}=\int_{-1}^{1} d q \int_{0}^{2 \pi} \sqrt{1-q^{2}} F_{1}(\varphi, q) \overline{F_{2}(\varphi, q)} d \varphi .
$$

Черта сверху означает комплексное сопряжение. Через $L^{2}\left(\mathbb{P}_{1}\right)$ обозначается гильбертово пространство комплекснозначных функций на $\mathbb{P}_{1}$ с тем же скалярным произведением (2.1). Функции из $L^{2}\left(\mathbb{P}_{1}\right)-$ это функции из $L^{2}\left(\widetilde{\mathbb{P}}_{1}\right)$, удовлетворяющие условию $F(\varphi+\pi,-q)=F(\varphi, q)$, и $L^{2}\left(\mathbb{P}_{1}\right) \hookrightarrow L^{2}\left(\widetilde{\mathbb{P}}_{1}\right)$. Следуя работе [28], в пространстве $L^{2}\left(\widetilde{\mathbb{P}}_{1}\right)$ можно ввести ортогональный базис $\left\{R_{l j}\right\}$ :

$$
\begin{gathered}
R_{l j}(\varphi, q)=e^{i l \varphi} U_{j}(q) \quad(l=0, \pm 1, \pm 2, \ldots, j=0,1,2, \ldots), \\
\left(R_{l j}, R_{l^{\prime} j^{\prime}}\right)_{L^{2}\left(\widetilde{\mathbb{P}}_{1}\right)}=\pi^{2} \delta_{j j^{\prime}} \delta_{l l^{\prime}},
\end{gathered}
$$


где $U_{j}(q)$ - полином Чебышева второго рода [29]. Пусть $\beta_{l j}(F)-$ коэффициенты Фурье функции $F \in L^{2}\left(\widetilde{\mathbb{P}}_{1}\right)$ относительно этого базиса. Введем основное для дальнейшего пространство функций $\{I\}, I: \mathbb{P}_{1} \rightarrow \mathbb{R}$. Это такие функции из $L^{2}\left(\mathbb{P}_{1}\right)$, что $I(\varphi, q)=2 \sqrt{1-q^{2}} F(\varphi, q)$ и $F \in L^{2}\left(\mathbb{P}_{1}\right)$. Для функций $I \in\{I\}$ коэффициенты $\alpha_{l j} \equiv \alpha_{l j}(I)$ определяются с помощью соотношения

$$
\alpha_{l j} \equiv \alpha_{l j}(I)=\beta_{l j}(F) \quad\left(I=2 \sqrt{1-q^{2}} F\right) .
$$

Функция $I: \widetilde{\mathbb{P}} \rightarrow \mathbb{R}$ принадлежит $\{I\}$, если $I(\varphi, q)=0$ при $|q|>1$ и

$$
\begin{gathered}
I(\varphi, q)=2 \sqrt{1-q^{2}} \sum_{l, j} \alpha_{l j} R_{l j}(\varphi, q), \\
\sum_{l, j}\left|\alpha_{l j}\right|^{2}<\infty, \quad \alpha_{l, j}=\bar{\alpha}_{-l, j}, \quad \alpha_{l j}=0, \text { если } l+j \text { нечетно. }
\end{gathered}
$$

Последнее условие следует из равенства $I(\varphi+\pi,-q)=I(\varphi, q)$. В работе [28] показано, что базис $\left\{R_{l j}\right\}$ (см. (2.2)) тесно связан с оператором Радона $\mathscr{R}$ (заданным формулой $(1.1))$ на $L^{2}(\mathbb{D})$. Фактически в этой работе доказана следующая теорема Пэли-Винера: функция $I: \mathbb{P}_{1} \rightarrow \mathbb{R}$ является преобразованием Радона некоторой функции $u \in L^{2}(\overline{\mathbb{D}})$ тогда и только тогда, когда $I \in\{I\}$ и выполняются условия

$$
\begin{gathered}
\sum_{l, j}\left|\alpha_{l j}\right|^{2}(j+1)<\infty, \\
\alpha_{l j} \equiv \alpha_{l j}(I)=0 \quad(|l|>j) .
\end{gathered}
$$

В соответствии с этим введем два ортогональных подпространства $\{J\} \subset\{I\}$ и $\left\{J^{\perp}\right\} \subset\{I\}$. По определению

$$
\begin{aligned}
& I \in\{J\}, \quad \text { если } \alpha_{l j}(I)=0 \text { при }|l|>j, \\
& I \in\left\{J^{\perp}\right\}, \quad \text { если } \alpha_{l j}(I)=0 \text { при }|l| \leqslant j .
\end{aligned}
$$

Тогда пространство $\{I\}$ разлагается в прямую сумму

$$
\{I\}=\{J\} \oplus\left\{J^{\perp}\right\}, \quad I=\Pi I+\Pi^{\perp} I .
$$

Здесь через $\Pi, \Pi^{\perp}$ обозначены ортогональные проекторы

$$
\Pi:\{I\} \rightarrow\{J\}, \quad \Pi^{\perp}:\{I\} \rightarrow\left\{J^{\perp}\right\} .
$$

Нам понадобятся также подпространства $\{I\}_{1} \subset\{I\},\{J\}_{1} \subset\{J\},\left\{J^{\perp}\right\}_{1} \subset\left\{J^{\perp}\right\}$ функций, удовлетворяющих условию (2.5). Согласно сказанному выше,

$$
\{I\}_{1}=\{J\}_{1} \oplus\left\{J^{\perp}\right\}_{1}
$$

и $\mathscr{R}^{-1}:\{J\}_{1} \rightarrow L^{2}(\overline{\mathbb{D}})$.

\section{§ 3. Формулировка результатов}

Основные результаты работы содержатся в теоремах 1, 2, сформулированных в этом параграфе. 
ТЕОРЕма 1 (формула обращения). Пусть $u \in L^{2}(\overline{\mathbb{D}}), z \in\{z\}_{m}, \mathscr{R}_{z} u=I$. Тогда можно указать такие числа $m_{0}, \varepsilon_{0}>0$, ито при выполнении для некоторого $m \geqslant m_{0}$ оценки

$$
\|z\|_{m} \leqslant \varepsilon \leqslant \varepsilon_{0}<1
$$

справедливы следующие соотношения:

I. Oператор $A_{z}=\mathscr{R}^{-1} \mathscr{R}_{z}-$ эллиптический НОФ нулевого порядка, допускающий расширение до почти унитарного обратимого оператора,

$$
\begin{array}{ll}
A_{z}: L^{2}\left(\mathbb{R}^{2}\right) \rightarrow L^{2}\left(\mathbb{R}^{2}\right), & \left\|A_{z}\right\|=1+O\left(\|z\|_{m}\right), \\
A_{z}^{T} A_{z}=\mathbb{I}+Q_{z}, & \left\|A_{z}^{T}\right\|=1+O\left(\|z\|_{m}\right), \\
A_{z}^{-1}=\left(\mathbb{I}+Q_{z}\right)^{-1} A_{z}^{T}, & \left\|A_{z}^{-1}\right\|=1+O\left(\|z\|_{m}\right),
\end{array}
$$

где $A_{z}^{T}$ - оператор, сопряженный $\kappa A_{z}$ в $L^{2}\left(\mathbb{R}^{2}\right), u$

$$
Q_{z}: L^{2}\left(\mathbb{R}^{2}\right) \rightarrow L^{2}\left(\mathbb{R}^{2}\right), \quad\left\|Q_{z}\right\| \leqslant C_{m}\|z\|_{m} .
$$

Более того, для любого $N$, такого, ито $3 \leqslant N \leqslant 2 m+2$, имеет место разложение

$$
Q_{z}=\sum_{n=1}^{N-1} Q_{n}+\Delta Q_{N}
$$

в котором $Q_{n}$ есть ПДО и

$$
\left\|Q_{n}\right\| \leqslant C_{n}\left(\|z\|_{m}\right)^{n}, \quad\left\|\Delta Q_{N}\right\| \leqslant C_{N}\left(\|z\|_{m}\right)^{N} .
$$

II. Oператор $\mathscr{R}_{z}$ обратим, и справедлива формула обращения

$$
u=\mathscr{R}_{z}^{-1} I, \quad \mathscr{R}_{z}^{-1}=\left(1+Q_{z}\right)^{-1} A_{z}^{T} \mathscr{R}^{-1} .
$$

ЗАМЕЧАНИЕ 1 . Числа $m_{0}, \varepsilon_{0}$ и зависящие только от $m$ константы в $O\left(\|z\|_{m}\right)$ можно явно указать. В частности, формула обращения (3.6) справедлива при $m_{0}=13$. Оператор $A_{z}$ в теореме 1 имеет вид

$$
A_{z} u(x)=\frac{1}{4 \pi^{2}} \int d \xi \int d y e^{i S(x, y, \xi)} a(x, y, \xi) u(y) .
$$

При этом фаза $S$ и символ $а$ определяется равенствами

$$
\begin{gathered}
S(x, y, \xi)=\langle y-x-z(y, \xi), \xi\rangle, \quad a(x, y, \xi)=a(y, \xi), \\
z(x, \xi)=z(\varphi, q, t), \quad \xi=|\xi| \eta,
\end{gathered}
$$

и переменные $x=\left(x_{1}, x_{2}\right)$ связаны с переменными $(q, t)$ соотношениями

$$
x=q \eta-t \eta^{*}+z(\varphi, q, t) .
$$

Символ $a(x, \xi)$ задается формулой

$$
a(x, \xi)=\frac{\left[1+|\partial z / \partial t|^{2}-2\left\langle\partial z / \partial t, \eta^{*}\right\rangle\right]^{1 / 2}}{1-\left\langle\partial z / \partial t, \eta^{*}\right\rangle+\left\langle\partial z / \partial q, \eta^{*}\right\rangle-[\partial z / \partial q, \partial z / \partial t]},
$$

где $[x, y]=x_{1} y_{2}-x_{2} y_{1}\left(x, y \in \mathbb{R}^{2}\right)$. Таким образом, в формуле $(3.7)$

$$
\begin{aligned}
a(x, y, \xi) & =a(y, \varphi), \quad z(x, \xi)=z(x, \varphi) & & (\xi=|\xi| \eta), \\
a(y, \xi) & =1+O\left(\|z\|_{1}\right), \quad a(y, \xi)=0 & & (y \geqslant 2) .
\end{aligned}
$$


Фаза $S^{T}$ и символ $a^{T}$ оператор $A_{z}^{T}$ также явно выписываются:

$S^{T}(x, y, \xi)=-S(y, x, \xi)=\langle y-x+z(x, \xi), \xi\rangle, \quad a^{T}(x, y, \xi)=a(y, x, \xi)=a(x, \xi)$.

ЗАмЕЧАНИЕ 2. В теореме 1 фактически содержится утверждение об обратимости ИОФ, фаза и символ которых удовлетворяют условиям (3.8), (3.11).

Чтобы сформулировать теорему 2 , введем оператор ограничения $\mathscr{D}^{\prime}$,

$$
\mathscr{D}^{\prime} f(x)= \begin{cases}f(x), & x \in \mathbb{D}^{\prime} \\ 0, & x \notin \mathbb{D}^{\prime}\end{cases}
$$

Здесь и ниже $\mathbb{D}^{\prime}$ - круг радиуса $1 / 2$.

ТЕОРема 2. Пусть выполняются условия на z из теоремы $1\left(\|z\|_{m} \leqslant \varepsilon \leqslant\right.$ $\left.\varepsilon_{0}<1 u m \geqslant m_{0}\right), u \in L^{2}\left(\mathbb{D}^{\prime}\right)$, supp $u \in \mathbb{D}^{\prime}$. Тогда

1) функция и $(x)$ однозначно восстанавливается по проекциии ПI (см. (2.8)) величины $I=\mathscr{R}_{z}$ и $и$

$$
\begin{aligned}
u & =B_{z} \mathscr{R}^{-1} \Pi I, \quad B_{z}: L^{2}\left(\mathbb{R}^{2}\right) \rightarrow L^{2}\left(\mathbb{D}^{\prime}\right), \\
B_{z} & =\mathscr{D}^{\prime} A_{z}^{-1}+\Delta B_{z}, \quad\left\|\Delta B_{z}\right\| \leqslant C_{m}\|z\|_{m} ;
\end{aligned}
$$

2) функизия $I(\varphi, q)=\mathscr{R}_{z} u(\varphi, q)$ удовлетворяет условиям Кавальери

$$
\Pi^{\perp} I=\Pi^{\perp} \mathscr{R}_{z}\left(B_{z} \mathscr{R}^{-1} \Pi I\right),
$$

u, таким образом, проекциия $\Pi^{\perp} I$ функциии $I=\mathscr{R}_{z}$ и определяется проекцией $\Pi I$.

ЗАмечАниЕ 3. Теорема 2 остается верной, если $\mathbb{D}^{\prime}-$ круг радиуса $r \leqslant 1-C$ $(C>0)$.

\section{§4. Доказательство теоремы 1}

4.1. Интегральный оператор Фурье $\boldsymbol{A}_{\boldsymbol{z}}$. Покажем, что оператор $A_{z}=\mathscr{R}^{-1} \mathscr{R}_{z}$ можно представить в виде (3.7) и при этом выполняются условия (3.8), (3.10), (3.11). Пусть $I=\mathscr{R}_{z} u$. По определению (1.2) оператора $\mathscr{R}^{-1}$

$$
A_{z} u(x)=\mathscr{R}^{-1} I(x)=\frac{1}{4 \pi^{2}} \int_{0}^{\infty} \lambda d \lambda \int_{0}^{2 \pi} e^{-i \lambda\langle x, \eta\rangle} \widehat{I}(\varphi, \lambda) d \varphi,
$$

а в силу определений (1.3), (1.6)

$$
\widehat{I}(\varphi, \lambda)=\int_{-1}^{1} e^{i \lambda q} d q \int_{-\sqrt{1-q^{2}}}^{\sqrt{1-q^{2}}} u(y(t))\left|\frac{d y}{d t}\right| d t
$$

Переходя в этом интеграле в соответствии с формулой $(3.9)$ от переменных $(q, t)$ к переменным $y=\left(y_{1}, y_{2}\right)=q \eta-t \eta^{*}+z(\varphi, q, t)$, получим, что

$$
\widehat{I}(\varphi, \lambda)=\int e^{i \lambda\langle y-x, \eta\rangle} u(y)|\mathscr{J}|^{-1}\left|\frac{d y}{d t}\right| d y
$$

и в этой формуле $\mathscr{J}-$ якобиан,

$$
\mathscr{J}=\left|\begin{array}{ll}
\partial y_{1} / \partial q & \partial y_{1} / \partial t \\
\partial y_{2} / \partial q & \partial y_{2} / \partial t
\end{array}\right|=-1+\left\langle\frac{\partial z}{\partial t}, \eta^{*}\right\rangle-\left\langle\frac{\partial z}{\partial q}, \eta\right\rangle+\left[\frac{\partial z}{\partial q}, \frac{\partial z}{\partial t}\right]
$$


Заметим, что отображение $(q, t) \rightarrow\left(y_{1}, y_{2}\right)$ переводит круг $q^{2}+t^{2} \leqslant 1$ в $\overline{\mathbb{D}}$ (так как $\left.\left.z\right|_{\partial \mathbb{D}}=0(1.5)\right)$ и взаимно однозначно. Подставляя выражение $\widehat{I}(\varphi, q)$ в (4.1) и переходя от переменных $(\varphi, \lambda)$ к переменным $\xi=\left(\xi_{1}, \xi_{2}\right)=\lambda \eta(\lambda=|\xi|)$, получим для оператора $A_{z}=\mathscr{R}^{-1} \mathscr{R}_{z}$ представление $(3.7)$, так как $a(y, \xi)=$ $|\mathscr{J}|^{-1}|d y / d t|$ (см. (3.10)). Докажем, что $A_{z}$ есть ИОФ нулевого порядка [30]. Замена переменных $\left(y_{1}, y_{2}\right) \leftrightarrow(q, t)$ почти ортогональна, и при $\|z\|_{m} \leqslant \varepsilon$ имеет место оценка

$$
\left|\partial_{x}^{n} z(x, \xi)\right| \leqslant C_{n}\|z\|_{n} .
$$

Учитывая, что $\varphi=\operatorname{arctg}\left(\xi_{2} / \xi_{1}\right)$ и $\left|\partial_{\xi}^{n} \varphi\right| \leqslant C_{n}|\xi|^{-n}$, получаем оценки

$$
\begin{aligned}
& \left|\partial_{\xi}^{n} z(x, \xi)\right| \leqslant C_{n}\|z\|_{n}|\xi|^{-n}, \\
& \left|\partial_{\xi}^{n} a(x, \xi)\right| \leqslant C_{n}\|z\|_{n+1}|\xi|^{-n} \quad(1 \leqslant n \leqslant m-1) .
\end{aligned}
$$

Следовательно, $\left|\partial_{\xi}^{\alpha} \partial_{x}^{\beta} \partial_{y}^{\gamma} a(x, y, \xi)\right| \leqslant C_{\alpha, \beta, \gamma}(1+|\xi|)^{-|\alpha|}$ при $|\alpha|+|\beta|+|\gamma| \leqslant m-1$ и $|\xi| \geqslant 1$. Непосредственно в силу определений (3.8), (3.11) $S(x, y, t \xi)=t S(x, y, \xi)$ и у фазы $S$ как функции от $y, \xi$ нет критических точек $\left(y^{c}, \xi^{c}\right)$ при $\xi^{c} \neq 0$. Таким образом, $A_{z}-$ эллиптический $(a(x, y, \xi) \geqslant C>0)$ ИОФ нулевого порядка.

4.2. Оператор $\boldsymbol{P}_{\boldsymbol{z}}$ и общая схема доказательства. В соответствии с общей идеологией теории ИОФ $[10,30]$ рассмотрим оператор $P_{z}=A_{z}^{T} A_{z}$. В нашем случае его ядро Шварца $K(x, y)$ легко вычисляется:

$$
K(x, y)=\frac{1}{4 \pi^{2}} \int e^{-i\langle y-x+z(x, \xi)-z(y, \xi), \xi\rangle} a(x, \xi) a(y, \xi) d \xi,
$$

и, следовательно,

$$
\begin{aligned}
P_{z} u(x) & =\frac{1}{4 \pi^{2}} \int d \xi \int d y e^{i \Phi(x, y, \xi)} p(x, y, \xi) u(y), \\
\Phi(x, y, \xi) & =\langle y-x+z(x, \xi)-z(y, \xi), \xi\rangle, \quad p(x, y, \xi)=a(x, \xi) a(y, \xi) .
\end{aligned}
$$

Задача состоит в том, чтобы доказать, что

$$
P_{z}=\mathbb{I}+Q_{z}, \quad\left\|Q_{z}\right\| \leqslant C_{m}\|z\|_{m} .
$$

Рассмотрим две функции $\rho_{0}(\xi), h(x, y)$, такие, что

$$
\begin{gathered}
\rho_{0}(\xi)=\rho_{0}(|\xi|)=\left\{\begin{array}{ll}
1, & |\xi| \leqslant 1, \\
0, & |\xi| \geqslant 2,
\end{array} \quad \rho_{0} \in C_{0}^{\infty},\left|\partial_{\xi}^{n} \rho_{0}(\xi)\right| \leqslant C_{n},\right. \\
h(x, y)=h(|x-y|)=\left\{\begin{array}{ll}
1, & |x-y| \leqslant 1, \\
0, & |x-y| \geqslant 2,
\end{array} \quad h \in C^{\infty},\left|\partial^{\alpha} h(x, y)\right| \leqslant C_{|\alpha|},\right.
\end{gathered}
$$

и определим функции $\tilde{\rho}(\xi), \tilde{h}(x, y)$ равенствами $\rho_{0}(\xi)+\tilde{\rho}(\xi)=1, h(x, y)+$ $\tilde{h}(x, y)=1$. Запишем оператор $P_{z}$ (см. (4.4)) в виде

$$
P_{z}=P_{z}^{0}+P_{z}^{1}+P_{z}^{2},
$$

где $P_{z}^{i}$ есть ИОФ с фазой $\Phi(x, y, \xi)$ (см. (4.4)) и символами $p_{z}^{i} \equiv p_{z}^{i}(x, y, \xi)$ $(i=0,1,2)$,

$$
\begin{gathered}
p_{z}^{0}(x, y, \xi)=\rho_{0}(\xi) p(x, y, \xi), \quad p_{z}^{1}(x, y, \xi)=\tilde{\rho}(\xi) \tilde{h}(x, y, \xi) p(x, y, \xi), \\
p_{z}^{2}(x, y, \xi)=\tilde{\rho}(\xi) h(x, y) p(x, y, \xi) .
\end{gathered}
$$


Введем операторы $\left.P_{0}^{i} \equiv P_{z}^{i}\right|_{z=0}$ и заметим, что по теореме Планшереля

$$
\sum_{i=0}^{2} P_{0}^{i}=\mathbb{I}
$$

Таким образом, для доказательства формулы (4.5) достаточно доказать, что

$$
P_{z}^{i}=P_{0}^{i}+Q_{z}^{i}, \quad\left\|Q_{z}^{i}\right\| \leqslant C_{m}\|z\|_{m} \quad(i=0,1,2) .
$$

Эти равенства являются определениями операторов $Q_{z}^{i}$, и будем оценивать их нормы $\left\|Q_{z}^{i}\right\|$ по отдельности.

4.3. Оператор $\boldsymbol{Q}_{\boldsymbol{z}}^{\mathbf{0}}$. В соответствии с определением (4.8)

$$
Q_{z}^{0} u(x)=\frac{1}{4 \pi^{2}} \int d \xi \int d y e^{i\langle y-x, \xi\rangle} q^{0}(x, y, \xi) u(y)
$$

и в этой формуле $q^{0}(x, y, \xi)=\rho_{0}(\xi) \Psi(x, y, \xi)$, где

$$
\Psi(x, y, \xi)=-1+p(x, y, \xi) e^{i\langle z(x, \xi)-z(y, \xi), \xi\rangle}
$$

и, следовательно,

$$
\Psi(x, y, \xi)=0 \text { при }|x| \geqslant 2 \text { и }|y| \geqslant 2 .
$$

Таким образом,

$$
Q_{z}^{0} u(x)=\int K^{0}(x, y) u(y) d y, \quad K^{0}(x, y)=\frac{1}{4 \pi^{2}} \int e^{i\langle y-x, \xi\rangle} q^{0}(x, y, \xi) d \xi
$$

Докажем оценку

$$
\left|K^{0}(x, y)\right| \leqslant C\|z\|_{13} \frac{\ln |x-y|+1}{|x-y|^{2}} \quad(|x-y| \geqslant 1) .
$$

Так как функция $z(x, \xi)$ негладкая в окрестности точки $\xi=0$, то разобьем область интегрирования по $\xi$ в интеграле $K^{0}(x, y)($ см. $(4.9))$ на две области $|\xi| \leqslant b^{-1}$ и $|\xi| \geqslant b^{-1}$, где $b=|x-y| \geqslant 1$. Этому соответствует представление $K^{0}=K_{1}^{0}+K_{2}^{0}$. Прямо по этому определению $\left|K_{1}^{0}\right| \leqslant C b^{-2} \max |\Psi|$, и так как $|\Psi| \leqslant C\|z\|_{0}$, то $\left|K_{1}^{0}\right| \leqslant C\|z\|_{0}|y-x|^{-2}$. Осталось рассмотреть величину

$K_{2}^{0}(x, y)=\frac{1}{4 \pi^{2}} \int_{b^{-1}}^{\infty} \lambda \rho_{0}(\lambda) d \lambda \int_{0}^{2 \pi} e^{-i \lambda b \cos (\varphi-\theta)} \Psi(x, y, \xi) d \varphi \quad(x-y=b(\cos \theta, \sin \theta))$.

Критические точки $\varphi_{c}=\theta, \pi+\theta$ фазы $\cos (\varphi-\theta)$ невырожденны, и, применяя к интегралу по $\varphi$ в (4.11) метод стационарной фазы с оценкой остаточного члена по теореме 2.1 из [31], получим

$$
\begin{aligned}
K_{2}^{0}(x, y)= & \frac{1}{4 \pi^{2}} \int_{b^{-1}}^{\infty} \lambda \rho_{0}(\lambda) e^{i \lambda b}\left[\frac{C_{1} g_{1}}{(\lambda b)^{1 / 2}}+\frac{C_{2} g_{2}}{(\lambda b)^{3 / 2}}\right] d \lambda+\Delta K_{2}^{0}(x, y), \\
& \left|\Delta K_{2}^{0}(x, y)\right| \leqslant C\|z\|_{1}(\ln |x-y|+1)|x-y|^{-2} .
\end{aligned}
$$

В этой формуле $g_{1} \equiv g_{1}(\lambda, x, y), g_{2} \equiv g_{2}(\lambda, x, y)$ - гладкие функции, содержащие производные от $z$ второго и четвертого порядков. Для завершения доказательства оценки (4.10) теперь достаточно интеграл в правой части формулы (4.12) оценить с помощью леммы Әрдейи [32]. Так как $\Psi=0$ при $|x|>2,|y|>2$, то нужная оценка

прямо следует из (4.10).

$$
\left\|Q_{z}^{0} u\right\| \leqslant C\|z\|_{13}\|u\|
$$


4.4. Оператор $\boldsymbol{Q}_{\boldsymbol{z}}^{\mathbf{1}}$. Согласно определению (см. (4.6), (4.7)),

$$
P_{z}^{1} u(x)=\int u(y) \tilde{h}(x, y) K_{z}^{1}(x, y) d y, \quad K_{z}^{1}(x, y)=\frac{1}{4 \pi^{2}} \int d \xi e^{i \Phi(x, y, \xi)} \tilde{\rho}(\xi) p(x, y, \xi) .
$$

Так как $\tilde{h}(x, y)=0$ при $|x-y| \leqslant 1$ и $\left|\partial \Phi / \partial \xi_{j}\right| \geqslant\left|y_{j}-x_{j}\right|-C\|z\|_{1}$, то к интегралу $K_{z}^{1}(x, y)$ можно применить формулу интегрирования по частям

$$
K_{z}^{1}(x, y)=\frac{i^{N}}{4 \pi^{2}} \int d \xi\left(F_{z}\right)^{N}[\tilde{\rho} p] e^{i \Phi},
$$

в которой оператор $F_{z}$ определяется равенством

$$
F_{z}[f]=\sum_{j} \partial_{j}\left(G^{-1}\left(\partial_{j} \Phi\right) f\right), \quad \partial_{j} \equiv \frac{\partial}{\partial \xi_{j}}, G \equiv\left|\nabla_{\xi} \Phi\right|^{2} .
$$

Из (4.3) следует, что

$$
\left|\partial_{\xi}^{n} \Phi\right| \leqslant C_{n} \lambda_{0} \lambda_{1}^{n} \quad(2 \leqslant n \leqslant 2 N), \quad \lambda_{0}=\|z\|_{2 N}|\xi|, \lambda_{1}=|\xi|^{-1},
$$

и мы будем использовать доказанную в [31] оценку

$$
\left|\left(F_{z}\right)^{N}[f]\right| \leqslant C_{N} \sum_{|\gamma|=0}^{N}\left|\partial_{\xi}^{n} f\right| \sum_{j=0}^{N} G^{-(N+j) / 2} \lambda_{0}^{j} \lambda_{1}^{N-|\gamma|+j} .
$$

В интересующем нас случае $f=\tilde{\rho} p$ и $G \geqslant C|x-y|^{2}$. Запишем выражение $\left(F_{z}\right)^{N}[f]$ в виде

$$
\left(F_{z}\right)^{N}[f]=\left(F_{0}\right)^{N}[f]+\Delta_{N} F_{z} f \quad\left(\left.F_{0} \equiv F_{z}\right|_{z=0}\right) .
$$

Тогда из (4.15) следует, что

$$
\left|\Delta_{N} F_{z} f\right| \leqslant C_{N}\|z\|_{2 N}|\xi|^{-N}|x-y|^{-N} .
$$

Введем обозначения $\left.\Phi^{0} \equiv \Phi\right|_{z=0}=\langle y-x, \xi\rangle, \Phi^{t}=\Phi^{0}+t\langle z(x, \xi)-z(y, \xi), \xi\rangle$ и определим величину $\Delta K_{z}^{1}(x, y)$ с помощью равенства

$$
K_{z}^{1}(x, y)=K_{0}^{1}(x, y)+\Delta K_{z}^{1}(x, y) \quad\left(\left.K_{0}^{1} \equiv K_{z}^{1}\right|_{z=0}\right) .
$$

Воспользуемся соотношениями

$$
\begin{aligned}
e^{i \Phi} & =e^{i \Phi^{0}}+i\langle z(x, \xi)-z(y, \xi), \xi\rangle \int_{0}^{1} e^{i \Phi^{t}} d t \\
K_{0}^{1}(x, y) & =\frac{i^{N}}{4 \pi^{2}} \int d \xi\left(F_{0}\right)^{N}[\tilde{\rho}] e^{i \Phi^{0}}=\frac{1}{4 \pi^{2}} \int d \xi \tilde{\rho}(\xi) e^{i\langle y-x, \xi\rangle} .
\end{aligned}
$$

Подставляя (4.19), (4.16) в (4.14) и учитывая неравенство (4.17), получим, что

$$
\left|\Delta K_{z}^{1}(x, y)\right| \leqslant C_{N}\|z\|_{2 N}|x-y|^{-N} \quad(\forall N \geqslant 4) .
$$

Теперь заметим, что, согласно определению (4.8) и соотношению (4.20),

$$
Q_{z}^{1} u(x)=\int \Delta K_{z}^{1}(x, y) \tilde{h}(x, y) u(y) d y .
$$

Выбирая $N=4$, имеем

$$
\left|Q_{z}^{1} u(x)\right| \leqslant C\|z\|_{8} \int|\tilde{h}(x, y)||u(y)||x-y|^{-4} d y,
$$


и отсюда сразу следует искомая оценка

$$
\left\|Q_{z}^{1} u\right\| \leqslant C\|z\|_{8}\|u\|
$$

4.5. Оператор $\boldsymbol{Q}_{\boldsymbol{z}}^{\mathbf{2}}$ и завершение доказательства. Рассмотрим оператор $P_{z}^{2}$ (см. (4.6), (4.7)). Перейдем от координат $\xi=\left(\xi_{1}, \xi_{2}\right)$ к координатам $\sigma=\left(\sigma_{1}, \sigma_{2}\right)$ :

$$
\sigma_{j}=\xi_{j}-\sum_{k} \frac{\partial z_{k}(x, \xi)}{\partial x_{j}} \xi_{k}
$$

В силу оценок (4.3) якобиан $|\partial \sigma / \partial \xi|$ этой замены при $|\xi| \geqslant 1$ удовлетворяет неравенству $|\partial \sigma / \partial \xi| \geqslant 1-C\|z\|_{2}$. Напомним, что $p_{z}^{2}(x, y, \xi)=0(|\xi| \leqslant 1)$ и, следовательно,

$$
\begin{gathered}
P_{z}^{2} u(x)=\frac{1}{4 \pi^{2}} \int d \sigma \int d y e^{i \Phi(x, y, \sigma)} \tilde{p}(x, y, \sigma) u(y), \\
\tilde{p}(x, y, \sigma)=\tilde{\rho}(\xi) h(x, y) p(x, y, \xi)\left|\frac{\partial \sigma}{\partial \xi}\right|^{-1}, \quad \Phi(x, y, \sigma)=\Phi(x, y, \xi(\sigma)) .
\end{gathered}
$$

Здесь и ниже в этом разделе $\xi=\xi(\sigma)$. По формуле Тейлора $\Phi(x, y, \sigma)=\langle y-x, \sigma\rangle+$ $\langle y-x, B(y-x)\rangle$, и матрица $B=\left(B_{j l}(x, y, \sigma)\right)$ определяется равенствами

$$
B_{j l}(x, y, \sigma)=-\frac{2}{j ! l !} \int_{0}^{1} d s(1-s)\left\langle\frac{\partial^{2} z}{\partial x_{j} \partial x_{l}}(x+s(y-z), \xi), \xi\right\rangle .
$$

Таким образом, $\Phi(x, y, \sigma)=\langle y-x, \xi\rangle+O\left(|x-y|^{2}|\xi|\right)$ и можно воспользоваться теоремой Хёрмандера [33], согласно которой $P_{z}$ является ПДО. При этом нам необходимо выделить из $P_{z}^{2}$ член $P_{0}^{2}$ и получить разложение величины $Q_{z}^{2}=$ $P_{z}^{2}-P_{0}^{2}$ по степеням $\partial^{\alpha} z$. Для этого достаточно аккуратно следовать схеме доказательства теоремы Хёрмандера. В результате получим

$$
P_{z}^{2} u(x)=\sum_{n=0}^{N-1} \frac{i^{n}}{n !}\left(Q_{z}^{2}\right)_{n} u(x)+\frac{i^{N}}{(N-1) !} \Delta_{N} P_{z}^{2} u(x)
$$

и в этом равенстве

$$
\begin{aligned}
\left(Q_{z}^{2}\right)_{n} u(x) & =\frac{1}{4 \pi^{2}} \int d \sigma \int d y e^{i\langle y-x, \sigma\rangle} q_{n}^{2}(x, y, \sigma) u(y) \\
q_{n}^{2}(x, y, \sigma) & =(-1)^{n} \sum_{\alpha_{1}, \beta_{1}, \ldots, \alpha_{n}, \beta_{n}} \frac{\partial^{2 n}}{\partial \sigma_{\alpha_{1}} \partial \sigma_{\beta_{1}} \ldots \partial \sigma_{\alpha_{n}} \partial \sigma_{\beta_{n}}} B_{\alpha_{1} \beta_{1}} \cdots B_{\alpha_{n} \beta_{n}} .
\end{aligned}
$$

Оператор $\Delta_{N} P_{z}^{2}$ в формуле (4.25) определяется следующим образом:

$$
\Delta_{N} P_{z}^{2} u(x)=\frac{1}{4 \pi^{2}} \int_{0}^{1}(1-t)^{N-2} d t \int d \sigma \int d y e^{i \Phi^{t}} q_{N}^{t} u .
$$


В этом равенстве $\Phi^{t} \equiv \Phi^{t}(x, y, \sigma)=\langle y-x, \sigma\rangle+t\langle y-x, B(y-x)\rangle$ и

$$
\begin{array}{r}
q_{N}^{t} \equiv q_{N}^{t}(x, y, \sigma)=(-1)^{N} \sum_{\substack{m_{1}, n_{1}, \ldots, m_{N}, n_{N} \\
\alpha_{1}, \beta_{1}, \ldots, \alpha_{N}, \beta_{N}}} \frac{\partial}{\partial \sigma_{n_{N}}}\left[G _ { \beta _ { N } n _ { N } } ^ { t } \frac { \partial } { \partial \sigma _ { m _ { N } } } \left[G_{\alpha_{N} m_{N}}^{t} \cdots\right.\right. \\
\cdots \frac{\partial}{\partial \sigma_{n_{1}}}\left[G_{\beta_{1} n_{1}}^{t} \frac{\partial}{\partial \sigma_{m_{1}}}\left[G_{\alpha_{1} m_{1}}^{t} B_{\alpha_{1} \beta_{1}} \cdots B_{\alpha_{N} \beta_{N}}\right] \cdots\right] .
\end{array}
$$

Остается определить величины $G_{k p}^{t}$. Так как

$$
\frac{\partial \Phi^{t}}{\partial \sigma_{p}}=y_{p}-x_{p}+t\left\langle y-x, \frac{\partial B}{\partial \sigma_{p}}(y-x)\right\rangle, \quad\left|\frac{\partial B_{j l}}{\partial \sigma_{p}}\right| \leqslant C\|z\|_{3}
$$

и по определению $\tilde{p}(x, y, \sigma)=0(|x-y| \geqslant 2)$, то можно считать, что $|x-y| \leqslant 2$. Тогда по теореме о неподвижной точке соотношение (4.28) разрешимо относительно $y_{p}-x_{p}$ и величины $G_{k p}^{t}$ определяются равенствами

$$
y_{k}-x_{k}=\sum_{p} G_{k p}^{t} \frac{\partial \Phi^{t}}{\partial \sigma_{p}} .
$$

Оператор $P_{0}^{2}$ выделим из оператора $\left(Q_{n}^{2}\right)_{0}$ (согласно формуле (4.25)). Для этого рассмотрим оператор $\left(Q_{n}^{2}\right)_{0}$ (см. (4.26)) и заметим, что $\left(Q_{n}^{2}\right)_{0} u(x)=P_{n}^{2} u(x)+$ $\Delta Q_{z 0}^{2} u(x)$, где $\Delta Q_{z 0}^{2}$ есть ПДО с символом $\Delta p_{z}^{0}(x, y, \sigma)=\tilde{p}(x, y, \sigma)-\tilde{\rho}(\xi) h(x, y)$. Оценим нормы операторов, входящих в разложение

$$
Q_{z}^{2}=\Delta Q_{z 0}^{2}+\sum_{n=1}^{N-1} \frac{i^{n}}{n !}\left(Q_{z}^{2}\right)_{n}+\frac{i^{N}}{(N-1) !} \Delta_{N} P_{z}^{2} .
$$

Из формул (4.26), (4.27) с учетом соотношений (4.29), (4.2), (4.3) получаем оценки

$$
\left|q_{n}^{2}(x, y, \sigma)\right| \leqslant C_{n}\left(\|z\|_{2 n+2}\right)^{n}|\xi|^{-n}, \quad\left|q_{N}^{t}(x, y, \sigma)\right| \leqslant C_{N}\left(\|z\|_{2 N+2}\right)^{N}|\xi|^{-N},
$$

из которых сразу следует, что при $3 \leqslant n \leqslant N-1$

$$
\left\|\left(Q_{z}^{2}\right)_{n}\right\| \leqslant C_{n}\left(\|z\|_{2 n+2}\right)^{n}, \quad\left\|\Delta_{N} P_{z}^{2}\right\| \leqslant C_{N}\left(\|z\|_{2 N+2}\right)^{N} .
$$

Для оценки норм собственных ПДО $\Delta Q_{z 0}^{2},\left(Q_{z}^{2}\right)_{n}(n=1,2)$ используем следующую оценку нормы собственного ПДО $A$ с символом $a \equiv a(x, y, \xi)\left(x, y, \xi \in \mathbb{R}^{2}\right)$ :

$$
\begin{gathered}
\|A\| \leqslant C \max _{\xi,|\alpha|=6}\left\{\|a\|_{1}+\left\|\partial^{\alpha} a\right\|_{1}\right\}, \quad\|a\|_{1} \equiv \int|a(x, y, \xi)| d x d y \\
\left(\partial^{\alpha} \equiv \partial_{x}^{\alpha_{1}} \partial_{y}^{\alpha_{2}},|\alpha|=\alpha_{1}+\alpha_{2}\right) .
\end{gathered}
$$

Эту оценку можно вывести, следуя доказательству теоремы 2.1 из книги [30], и с помощью нее получаем

$$
\left\|\Delta Q_{z 0}^{2}\right\| \leqslant C\|z\|_{7}, \quad\left\|\left(Q_{z}^{2}\right)_{n}\right\| \leqslant C\left(\|z\|_{2 n+8}\right)^{n} \quad(n=1,2) .
$$

Выбирая $N=3$ в (4.30), приходим к неравенству $\left\|Q_{z}^{2}\right\| \leqslant C\|z\|_{12}$. Эта оценка вместе с (4.13) и (4.21) завершает доказательство утверждений (3.2), (3.3) и формулы обращения (3.6) при $m \geqslant 13$. Для доказательства утверждений (3.4), (3.5) теперь достаточно воспользоваться разложением (4.30) и оценками (4.31), (4.32). Теорема 1 доказана. 


\section{§5. Обратный оператор Радона на пространстве $\{I\}_{1}$}

Рассмотрим действие оператора $\mathscr{R}^{-1}$ (см. (1.2)) на пространстве $\{I\}_{1}$, определенном формулой (2.10). Если ввести функции $I_{l j}(\varphi, q), f_{l j}(x)$ равенствами

$$
I_{l j}(\varphi, q)=\left\{\begin{array}{ll}
2 \sqrt{1-q^{2}} R_{l j}(\varphi, q), & |q| \leqslant 1, \\
0, & |q|>1,
\end{array} \quad f_{l j}(x)=\left(\mathscr{R}^{-1} I_{l j}\right)(x),\right.
$$

то, как показано в [28], при $j \geqslant|l|, j=|l|+2 s$ ( $s$ целое)

$$
f_{l j}(x)= \begin{cases}(j+1) Z_{l s}(x), & |x| \leqslant 1 \\ 0, & |x|>1\end{cases}
$$

Здесь $Z_{l s}(x)$ - полином Цернике [34,35],

$$
\begin{gathered}
Z_{l s}(x)=e^{i l \theta} r^{|l|} P_{s}^{(0,|l|)}\left(2 r^{2}-1\right) \quad(l=0, \pm 1, \pm 2, \ldots, s=0,1,2, \ldots), \\
x=r(\cos \theta, \sin \theta),
\end{gathered}
$$

где $P_{n}^{(\alpha, \beta)}-$ полином Якоби. Система полиномов Цернике ортогональна и полна в $L^{2}(\mathbb{D})$,

$$
\int_{\mathbb{D}} Z_{l s}(x) Z_{l^{\prime} s^{\prime}}(x) d x=\pi(|l|+2 s+1)^{-1} \delta_{l l^{\prime}} \delta_{s s^{\prime}} .
$$

Таким образом, определено действие оператора $\mathscr{R}^{-1}$ на $\{J\}_{1}$ и осталось рассмотреть действие этого оператора на $\left\{J^{\perp}\right\}_{1}$ (см. (2.10)).

Лемма. Если $|l|>j,|l|=j+2 s$ u $s \geqslant 1-$ целое число, то

$$
f_{l j}(x)= \begin{cases}0, & r \equiv|x|<1, \\ (-1)^{s}(j+1) r^{-j-2} P_{s-1}^{(j+1,0)}\left(1-2 / r^{2}\right), & r \geqslant 1,\end{cases}
$$

и эти функции ортогональны в $L^{2}\left(\mathbb{R}^{2}\right)$ :

$$
\left(f_{l j}, f_{l^{\prime} j^{\prime}}\right)=\pi \delta_{l l^{\prime}} \delta_{j j^{\prime}}
$$

ДокАЗАТЕЛЬСтво. Непосредственно из определения (5.1) функции $I_{l j}$ следует, что при любых $l, j$

$$
\widehat{I}_{l j}(\varphi, \lambda)=2 \pi e^{i \pi j / 2}(j+1) \lambda^{-1} J_{j+1}(\lambda),
$$

где $J_{\nu}(t)$ - функции Бесселя. Вывод этой формулы по существу содержится в [34]. Используя формулу (5.5) и определение $(1.2)$ оператора $\mathscr{R}^{-1}$, заключаем, что при любых $l, j$

$$
f_{l j}(x)=e^{i l \theta}(j+1) e^{i \pi(j-|l|) / 2} \int_{0}^{\infty} J_{j+1}(\lambda) J_{|l|}(\lambda r) d \lambda .
$$

Интеграл по $\lambda$ в этой формуле является табличным [36], и это дает равенства (5.2), (5.3). С помощью равенства (5.5) и формулы Парсеваля получаем

$$
\left(f_{l j}, f_{l j^{\prime}}\right)=2 \pi \delta_{l l^{\prime}} e^{i \pi\left(j-j^{\prime}\right) / 2}(j+1)\left(j^{\prime}+1\right) \int_{0}^{\infty} \lambda^{-1} J_{j+1}(\lambda) J_{j^{\prime}+1}(\lambda) d \lambda .
$$


Для доказательства формулы (5.4) теперь достаточно заметить, что нас интересует случай, когда $j-j^{\prime}-$ четное число и (см. [37])

$$
\int_{0}^{\infty} x^{-1} J_{\mu}(x) J_{\nu}(x) d x=\frac{2}{\pi\left(\nu^{2}-\mu^{2}\right)} \sin \frac{\nu-\mu}{2} \pi .
$$

Лемма доказана.

Из этой леммы следует, что $\mathscr{R}^{-1} \Pi$ и $\mathscr{R}^{-1} \Pi^{\perp}-$ ограниченные операторы из $\{I\}_{1}$ в $L^{2}\left(\mathbb{R}^{2}\right)$ и

$$
\mathscr{R}^{-1} \Pi=\mathscr{D} \mathscr{R}^{-1}, \quad \mathscr{R}^{-1} \Pi^{\perp}=\mathscr{D}^{\perp} \mathscr{R}^{-1},
$$

где $\mathscr{D}$ и $\mathscr{D}^{\perp}\left(\mathscr{D}+\mathscr{D}^{\perp}=\mathbb{I}\right)-$ операторы ограничения,

$$
\mathscr{D} f(x)=\left\{\begin{array}{ll}
f(x), & x \in \mathbb{D}, \\
0, & x \notin \overline{\mathbb{D}},
\end{array} \quad \mathscr{D}^{\perp} f(x)= \begin{cases}0, & x \in \mathbb{D}, \\
f(x), & x \notin \overline{\mathbb{D}} .\end{cases}\right.
$$

\section{§6. Доказательство теоремы 2}

По предположению $u(x)=0$ при $|x|>1 / 2$ и, следовательно, $\mathscr{R}_{z} u(\varphi, q)=0$ при $|q|>1 / 2+\|z\|_{0}$. Для доказательства включения $\mathscr{R}_{z} u \in\{I\}$, таким образом, достаточно доказать, что $\mathscr{R}_{z} u \in L^{2}\left(\mathbb{P}_{1}\right)$. Это сразу следует из определения (1.6) ОПР $\mathscr{R}_{z} u$, так как неравенство Шварца дает оценку $\left\|\mathscr{R}_{z} u\right\|_{L^{2}\left(\widetilde{\mathbb{P}}_{1}\right)} \leqslant C\left(1+\|z\|_{1}\right)\|u\|$. Теперь $I=\mathscr{R}_{z} u \in\{I\}$, и разложение $I=\Pi I+\Pi^{\perp} I$ из (2.8) позволяет записать уравнение $I=\mathscr{R}_{z} u$ в виде двух уравнений

$$
\begin{aligned}
\Pi \mathscr{R}_{z} u & =\Pi I, \\
\Pi^{\perp} \mathscr{R}_{z} u & =\Pi^{\perp} I .
\end{aligned}
$$

Если доказать обратимость оператора $П \mathscr{R}_{z}$ на его образе, то уравнение (6.2) и даст условия Кавальери. Подчеркнем, что существование решения $u$ предполагается. Так как $\operatorname{Ker} \mathscr{R}^{-1}=0$, то, применяя оператор $\mathscr{R}^{-1}$ к обеим частям уравнения (6.1), получим, что $\mathscr{R}^{-1} \Pi \mathscr{R}_{z} u=g, g \equiv \mathscr{R}^{-1} \Pi I$. Если предположить, что $g \in L^{2}\left(\mathbb{R}^{2}\right)$, то в силу формул (5.7) речь идет о решении граничной задачи $\mathscr{D} A_{z} u=g$. Так как $A_{z} u=g+\mathscr{R}^{-1} \Pi^{\perp} \mathscr{R}_{z} u$, то

$$
u=A_{z}^{-1} g+A_{z}^{-1} \Psi, \quad \Psi(x)=\mathscr{R}^{-1} \Pi^{\perp} \mathscr{R}_{z} u .
$$

Оценим норму $\|\Psi\|$. В силу формулы (5.4) имеем $\|\Psi\|^{2}=\pi \sum_{|l|>j}\left|\alpha_{l j}(I)\right|^{2}(j+1)$ и, следовательно,

$$
\|\Psi\|^{2} \leqslant C \sum_{|l| \geqslant 1}(|l|+1)^{2}\left(\max _{j<|l|}\left|\alpha_{l j}(I)\right|\right)^{2},
$$

где коэффициенты $\alpha_{l j} \equiv \alpha_{l j}(I)$ определены в формулах (2.3), (2.4) и

$$
\alpha_{l j}=\frac{1}{2 \pi^{2}} \int_{0}^{2 \pi} d \varphi \int_{-1}^{1}\left(\mathscr{R}_{z} u\right)(\varphi, q) \bar{R}_{l j}(\varphi, q) d q .
$$


Подставляя сюда выражение (1.6) для $\mathscr{R}_{z}$ и переходя от переменных $(q, t)$ к переменным $x=\left(x_{1}, x_{2}\right)$, согласно $(3.9)$, получим

$$
\begin{aligned}
\alpha_{l j} & =\frac{1}{2 \pi^{2}} \int_{\mathbb{D}^{\prime}} u(x) A_{l j}(x) d x, \\
A_{l j}(x) & =\int_{0}^{2 \pi} e^{-i l \varphi} U_{j}(\langle x, \eta\rangle-\langle z(x, \varphi), \eta\rangle) a(x, \varphi) d \varphi,
\end{aligned}
$$

где $a(x, \varphi)$ - символ оператора $A_{z}^{T}$, определенный формулой $(3.10)$. Введем следующие функции $g, h: g(x, \varphi)=a(x, \varphi)-1, h(x, \varphi)=\langle z(x, \varphi), \eta\rangle$. Используя тождество

$$
U_{j}(\langle x, \eta\rangle-h)=U_{j}(\langle x, \eta\rangle)-h \int_{0}^{1} U_{j}^{(1)}(\langle x, \eta\rangle-s h) d s
$$

получим, что

$$
\begin{aligned}
& A_{l j}(x)=\int_{0}^{2 \pi} e^{-i l \varphi} U_{j}(\langle x, \eta\rangle) d \varphi+\int_{0}^{2 \pi} e^{-i l \varphi} F_{j}(\varphi) d \varphi \\
& F_{j}(\varphi) \equiv F_{j}(\varphi, x)=g U_{j}(\langle x, \eta\rangle)-h(1+g) \int_{0}^{1} U_{j}^{(1)}(\langle x, \eta\rangle-s h) d s .
\end{aligned}
$$

Так как нас интересует случай $|l|>j$, то первый интеграл в правой части равенства (6.6) равен нулю. Используя определение $U_{j}(t)=\left(1-t^{2}\right)^{-1 / 2} \sin (j+1) \arccos t$, сводим оценку величин $A_{l j}(x)$ к оценке осциллирующих интегралов вида

$$
\widetilde{A}_{l j}(x)=\int_{0}^{2 \pi} e^{i S(\varphi)} f_{j}(\varphi) d \varphi
$$

с фазой $S(\varphi)=-l \varphi \pm(j+1) \arccos (\langle x, \eta\rangle-s h)$. Нас интересует случай $j+1 \leqslant|l|$, $|x| \leqslant 1 / 2$. При этих условиях фаза $S(\varphi)$ не имеет критических точек и $\left|S^{(1)}(\varphi)\right| \geqslant$ $C|l|$. Интегрируя по частям и используя оценку $(4.15)$, получим, что $\left|A_{l j}(x)\right| \leqslant$ $C_{N}\|z\|_{N+1}\left(1+\|z\|_{N}\right)^{N}|l|^{-N}$ при любом $N \geqslant 0$ и в силу равенства (6.5) при $|l|>j$

$$
\left|\alpha_{l j}\right| \leqslant C_{N}\|z\|_{n+1}\left(1+\|z\|_{N}\right)^{N}\|u\|_{N}|l|^{-N} .
$$

Подставляя эту оценку в (6.4), имеем

$$
\|\Psi\| \leqslant C_{N}\|z\|_{N+1}\|u\| \quad(2 \leqslant N \leqslant m-1) .
$$

Так как $u=\mathscr{D}^{\prime} u$, где $\mathscr{D}^{\prime}$ - оператор ограничения на $\mathbb{D}^{\prime}$, то уравнение $(6.3)$ можно записать в виде $u=\mathscr{D}^{\prime} A_{z}^{-1} g+\mathscr{D}^{\prime} G_{z} u, G_{z} \equiv A_{z}^{-1} \mathscr{R}^{-1} \Pi^{\perp} \mathscr{R}_{z}$. Из (6.7) и (3.2) следует, что на $L^{2}\left(\mathbb{D}^{\prime}\right)$

$$
\left\|\mathscr{D}^{\prime} G_{z}\right\| \leqslant C_{N}\|z\|_{N+1} \quad(2 \leqslant N \leqslant m-1) .
$$

Выбирая $N$ из условия $2 \leqslant N \leqslant 12$, получим, что оператор $1-\mathscr{D}^{\prime} G_{z}$ обратим и для решения $u$ уравнения (6.1) или (6.3) имеет место равенство

$$
u=\left(1-\mathscr{D}^{\prime} G_{z}\right)^{-1} \mathscr{D}^{\prime} A_{z}^{-1} T_{z} u=B_{z} \mathscr{R}^{-1} \Pi I,
$$

где $T_{z}=\mathscr{R}^{-1} \Pi \mathscr{R}_{z}=\mathscr{D} A_{z}$ и $B_{z} T_{z}=\mathbb{I}$ на $L^{2}\left(\mathbb{D}^{\prime}\right)$. Это и дает нужную формулу (3.12) восстановления функции $u \in L^{2}\left(\mathbb{D}^{\prime}\right)$ по проекции $\Pi I=\Pi \mathscr{R}_{z} u$ вместе с нужной оценкой нормы оператора $\Delta B_{z}$ в (3.13). Подставляя формулу восстановления в (6.2), получим условия Кавальери (3.14).

Теорема 2 доказана. 


\section{ЛИТЕРАТУРА}

1. Гельфанд И. М., Граев М. И., Виленкин Н. Я. Интегральная геометрия и связанные с ней вопросы теории представлений. Обобщенные функции, вып. 5. Физматгиз, М., 1967.

2. Гельфанд И. М., Гиндикин С. Г., Граев М. И. Избранные задачи интегральной геометрии. Добросвет, М., 2000.

3. Хелгасон С. Преобразование Радона. Мир, М., 1983.

4. Хелгасон C. Группы и геометрический анализ. Мир, М., 1987.

5. Гельфанд И. М., Граев М. И., Шапиро З. Я. Дифференциальные формы и интегральная геометрия. Функц. анализ и его прил., 3, вып. 2, 24-40 (1969).

6. Guillemin $V$. On some results of Gelfand in integral geometry. In: Pseudodifferential operators and Applications (Notre Dame, Ind., 1984), Proc. Sympos. Pure Math., Vol. 43, Providence, R.I., 1985, pp. 149-155.

7. Гийемин В., Стернберг C. Геометрические асимптотики. Мир, М., 1981.

8. Quinto E. T. The dependence of the generalized Radon transform on defining measures. Trans. Amer. Math. Soc., 257, No. 2, 331-346 (1980).

9. Greenleaf A., Uhlmann $G$. Nonlocal inversion formulas for the $X$-ray transformation. Duke Math. J., 58, No. 1, 205-240 (1989).

10. Stein E. M. Harmonic Analysis: Real Variable Methods, Orthogonality, and Oscillatory Integrals. Princeton University Press, Princeton, 1993.

11. Phong D. H. Regularity of Fourier integral operators. In: Proc. Intern. Congress of Math., Zurich, 1994, Vol. 2, Birkhauser, Basel, 1995, pp. 862-874.

12. Phong D. H., Stein E. M. Singular Radon transforms and oscillatory integrals. Duke Math. J., 58, No. 2, 347-369 (1989).

13. Quinto E. T. Radon transforms on curves in the plane. In: Thomography, Impedance Imaging, and Integral Geometry. Lectures in Appl. Math., Vol. 30, Amer. Math. Soc., Providence, R.I., 1994, pp. 231-244.

14. Boman J., Quinto E. T. Support theorems for real-analytical Radon transforms. Duke Math. J., 55, No. 4, 943-948 (1987).

15. Agranousky M. L., Quinto E. T. Injectivity sets for the Radon transform over circles and complete systems of radial functions. J. Funct. Anal., 139, 383-414 (1996).

16. Greenleaf A., Uhlmann $G$. Estimates for singular Radon transforms and pseudodifferential operators with singular symbols. J. Funct. Anal., 89, 202-239 (1990).

17. Thomson A. A. Sobolev estimates for singular Radon transforms. J. Funct. Anal., 112, 61-96 (1993).

18. Йон Ф. Плоские волны и сферические средние в приложении к дифференциальным уравнениям с частными производными. Мир, М., 1958.

19. Kурант P.Уравнения с частными производными. Мир, М., 1964.

20. Лаврентьев М. М., Васильев В. Г., Романов В. Г. Многомерные обратные задачи для дифференциальных уравнений. Наука, Новосибирск, 1969.

21. Романов В. Г. Восстановление функции через интегралы по семейству кривых. Сиб. матем. ж., 7, № 5, 1206-1208 (1967).

22. Cormack A. M. Radon transform on a family curves in the plane, I. Proc. Amer. Math. Soc., 83, 325-330 (1981); II, ibid., 86, 293-298 (1982).

23. Lax P. D., Phillips P. S. The Paley-Wiener theorem for the Radon transform. Comm. Pure Appl. Math., 23, No. 3, 409-424 (1970). [Имеется перевод: Математика, 16:3, 97-112 (1972).]

24. Мухометов Р. Г. О задаче интегральной геометрии. В кн.: Математические проблемы геофизики, вып. 6, Новосибирск, 1975, с. 212-242.

25. Аниконов Ю. Е. О разрешимости одной задачи интегральной геометрии. Матем. сб., 101 (143), № 2, 271-279 (1976).

26. Мухометов Р. Г. Задача восстановления двумерной римановой метрики и интегральная геометрия. ДАН СССР, 232, № 1, 32-35 (1977). 
27. Бухгейм А. Л. Введение в теорию обратных задач. Наука, Новосибирск, 1988.

28. Marr R. B. On the reconstruction of a function on a circular domain from a sampling of its line integrals. J. Math. Anal. Appl., 45, 357-374 (1974).

29. Суәтин П. К. Ортогональные полиномы. Наука, М., 1979.

30. Трев $\Phi$. Введение в теорию псевдодифференциальных операторов и интегральных операторов Фурье. Т. 1, 2, Мир, М., 1984.

31. Попов Д. А. Оценки с константами для некоторого класса осциллирующих интегралов. УМН, 52, вып. 1, 77-148 (1997).

32. Эрдейи A. Асимптотические разложения. Физматгиз, М., 1962.

33. Hörmander $L$. The spectral function of elliptic operator. Acta Math., 121, Nos. 3-4, 193-218 (1968). [Имеется перевод: Математика, 13:6, 114-137 (1969).]

34. Deans R. S. The Radon Transform and Some Its Applications. John Wiley and Sons, New York, 1983.

35. Борн М., Вольф Э. Основы оптики. Наука, М., 1973.

36. Бейтмен Г., Эрдейи A. Таблицы интегральных преобразований. Т. 2, Наука, М., 1970.

37. Прудников А. П., Брычков Ю. А., Маричев О. И. Интегралы и ряды специальных функций. Наука, М., 1983.

Московский государственный университет, Институт физико-химической биологии

Поступило в редакцию им. А. Н. Белозерского 20 марта 2001 г. 